\title{
NLS breathers, rogue waves, and solutions of the Lyapunov equation for Jordan blocks
}

\author{
Oleksandr Chvartatskyi and Folkert Müller-Hoissen \\ Max-Planck-Institute for Dynamics and Self-Organization, Am Fassberg 17, 37077 Göttingen, Germany \\ E-mail: alex.chvartatskyy@gmail.com, folkert.mueller-hoissen@ds.mpg.de
}

\begin{abstract}
The infinite families of Peregrine, Akhmediev and Kuznetsov-Ma breather solutions of the focusing Nonlinear Schrödinger (NLS) equation are obtained via a matrix version of the Darboux transformation, with a spectral matrix of the form of a Jordan block. The structure of these solutions is essentially determined by the corresponding solution of the Lyapunov equation. In particular, regularity follows from properties of the Lyapunov equation.
\end{abstract}

\section{Introduction}

In 1983 Howell Peregrine [1] showed that the "self-focusing" Nonlinear Schrödinger (NLS) equation

$$
\mathrm{i} \mathfrak{q}_{t}+\mathfrak{q}_{x x}+2|\mathfrak{q}|^{2} \mathfrak{q}=0
$$

admits an exact solution that models a rogue wave (or "freak wave"), i.e., a wave with a significant amplitude that seems to "appear from nowhere" and "disappears without a trace" (cf. [2]). The existence of this Peregrine breather is due to the modulational (Benjamin-Feir) instability of the background solution $e^{2 \mathrm{it}}$ on which it emerges. Up to this factor, it is given by a rational expression,

$$
\mathfrak{q}=\left(1-\frac{G+\mathrm{i} H}{D}\right) e^{2 \mathrm{i} t}
$$

where $D, G, H$ are real polynomials in the independent variables $x$ and $t$. That this "instanton" solution indeed models physical phenomena, has been demonstrated recently in nonlinear fibre optics [3], for deep water waves [4], and in a multicomponent plasma [5] 1. Also see [6, 7], for example.

The Peregrine breather is the analog of an NLS soliton, on the nontrivial background and for a special value of a spectral parameter. One can superpose an arbitrary number of such solutions in a nonlinear way. This includes an infinite family of exact solutions, where the $n$th member arises from a nonlinear superposition of $n$ Peregrine breathers, by taking a limit where associated spectral parameters tend to a special value. These solutions have the above quasi-rational form. The next to Peregrine member of this family apparently appeared first in [8]. It has also been shown to model certain waves observed in a water tank 9 .

To construct this family of solutions, the authors of [2] started from a linear system (Lax pair) for the focusing NLS equation, with a specific choice of the spectral parameter. Solutions are then constructed using Darboux transformations 10,11. The authors of [2] met the problem that the usual iteration of Darboux transformations requires a different spectral parameter at each step. But addressing quasi-rational solutions, one has to use the same spectral parameter, and this

\footnotetext{
${ }^{1}$ In some of these physical models, $x$ and $t$ as spatial and time variables are exchanged in 1.1 .
} 
requires some modifications of the usual scheme. A corresponding improvement of the Darboux transformation method has been formulated in [12]. An even richer family of quasi-rational solutions than obtained in [2] has been provided in [13 15], expressing them as quotients of Wronskians. Via Hirota's bilinear method such solutions have been derived in [16].

Our present work uses yet another variant of the Darboux method. We use a single, but vectorial Darboux transformation and a linear system that involves a spectral parameter matrix $Q$. This linear system is "degenerate" for a special eigenvalue of $Q$. The $n$th order quasi-rational solution of the focusing NLS equation is then obtained by choosing for $Q$ an $n \times n$ Jordan block with this special eigenvalue. This yields a nice characterization of the generalizations of the Peregrine breather. The vectorial Darboux transformation does not meet the problems mentioned in [2] and does not require modifications or generalizations as in $[12$.

Shortly after Peregrine's publication, a solution [17] of the NLS equation was found which is also localized in "time" $t$. But in contrast to the Peregrine breather, it is periodic in $x$-direction. This Akhmediev breather yields the Peregrine breather in a certain limit. Also the Akhmediev breather belongs to an infinite family of exact solutions.

Morover, there is a counterpart of the Akhmediev breather that is localized in $x$-direction and periodic in $t$-direction. It is known as Kuznetsov-Ma breather 18, 19 and has been observed in fibre optics [20]. The Akhmediev and Kuznetsov-Ma breathers can be combined into a common expression, from which we recover one or the other if the modulus of a spectral parameter is below or above a certain value. The Peregrine breather is then obtained for the transition value, the abovementioned special eigenvalue of $Q$. Both, the Akhmediev and the Kuznetsov-Ma breather, have meanwhile been observed in water tank experiments [21]. Like the Peregrine breather, also the Akhmediev-Kuznetsov-Ma (AKM) breather belongs to an infinite sequence of exact solutions with increasing complexity.

Whereas regularity of the generated solution is an easy problem in case of a single scalar Darboux transformation for the NLS equation, it is more involved in case of a vectorial Darboux transformation. But one can use properties of the Lyapunov equation (see the Appendices) to solve this problem satisfactorily. Moreover, the main purpose of this work is to show that the structure of the members of the families of Akhmediev, Kuznetsov-Ma and Peregrine breathers is largely determined by the solution of the rank one Lyapunov equation with a Jordan block matrix.

In Section 2 we recall a Darboux transformation result for the focusing NLS equation. This is then elaborated in Section 3 for the abovementioned seed solution. Section 4 contains concluding remarks.

\section{Darboux transformation for the focusing NLS equation}

Let $\phi$ be a $2 \times 2$ matrix subject to the AKNS equation

$$
\mathrm{i}\left[J, \phi_{t}\right]+2 \phi_{x x}-\left[[J, \phi], \phi_{x}\right]=0,
$$

where $J=\operatorname{diag}(1,-1)$. Decomposing $\phi$ as follows,

$$
\phi=J\left(\begin{array}{ll}
u & \mathfrak{q} \\
\mathfrak{r} & v
\end{array}\right)=\left(\begin{array}{rr}
u & \mathfrak{q} \\
-\mathfrak{r} & -v
\end{array}\right)
$$

and imposing the reduction condition

$$
\phi^{\dagger}=\phi,
$$

where $^{\dagger}$ indicates Hermitian conjugation, implies that $u$ and $v$ are real, $\mathfrak{r}=-\mathfrak{q}^{*}$ (where ${ }^{*}$ denotes complex conjugation), and (2.1) becomes

$$
\mathrm{i} \mathfrak{q}_{t}+\mathfrak{q}_{x x}+\mathfrak{q} v_{x}+u_{x} \mathfrak{q}=0, \quad\left(u_{x}-|\mathfrak{q}|^{2}\right)_{x}=0, \quad\left(v_{x}-|\mathfrak{q}|^{2}\right)_{x}=0
$$


Setting possible constants of integration (actually functions of $t$ ) to zero by integrating the last two equations, and eliminating $u_{x}$ and $v_{x}$ in the first equation, we obtain the focusing NLS equation (1.1). Any solution of the a priori more general system 2.4 can be transformed into a solution of the focusing NLS equation (cf. [22], for example).

The following Darboux transformation is obtained from a general binary Darboux transformation result 23] in bidifferential calculus, see 24.

Proposition 2.1. Let $\phi_{0}$ be a solution of (2.1) and (2.3), $Q$ a constant $n \times n$ matrix satisfying the spectrum condition

$$
\sigma(Q) \cap \sigma\left(-Q^{\dagger}\right)=\emptyset
$$

and $\eta$ an $n \times 2$ matrix solution of the linear system

$$
\mathrm{i} \eta_{t}=-Q \eta_{x}+\eta \phi_{0, x}, \quad \eta_{x}=-\frac{1}{2} Q \eta J-\frac{1}{2} \eta\left[J, \phi_{0}\right]
$$

Furthermore, let $\Omega$ be an $n \times n$ matrix solution of the Lyapunov equation

$$
Q \Omega+\Omega Q^{\dagger}=\eta \eta^{\dagger} .
$$

Then, wherever $\Omega$ is invertible,

$$
\phi=\phi_{0}-\eta^{\dagger} \Omega^{-1} \eta
$$

is a new solution of (2.1) and (2.3). As a consequence, the components of $\phi$ yield a solution of (2.4).

(2.6) is a Lax pair for (2.1), i.e., 2.1) is the compatibility condition arising from $\eta_{x t}=\eta_{t x}$. Note that $Q$ plays the role of a matrix spectral parameter.

Solutions of (2.1) obtained via Proposition 2.1 in this work indeed yield via (2.2) directly solutions $\mathfrak{q}$ of the focusing NLS equation (1.1). This is so because we start from a seed solution that satisfies the focusing NLS equation and which determines the asymptotics of the generated solutions.

Remark 2.2. Dropping the spectrum condition 2.5) in Proposition 2.1. we need to add the following two equations

$$
\Omega_{x}=-\frac{1}{2} \eta J \eta^{\dagger}, \quad \mathrm{i} \Omega_{t}=\frac{1}{2}\left(Q \eta J \eta^{\dagger}-\eta J \eta^{\dagger} Q^{\dagger}\right)+\frac{1}{2} \eta\left[J, \phi_{0}\right] \eta^{\dagger},
$$

which otherwise follow by differentiation of $(2.7)$ and application of the remaining equations. Using these equations, (2.6), 2.7) and the assumption that $\phi_{0}$ solves (2.1), one can also prove the proposition by a direct but lengthy computation.

Remark 2.3. The (algebraic) Lyapunov equation plays an important role in the stability analysis of systems of ordinary differential equations (see, e.g., 25]). It is a special case of Sylvester's equation 26. An appearance of the latter in a Riemann-Hilbert factorization problem dates back to 1986 27. Sylvester's equation typically enters the stage when matrix (or operator) versions of integrable (differential or difference) equations are considered [28], or when matrix methods are applied, e.g., to concisely express iterated Bäcklund or Darboux transformations (see, e.g., 29 and references therein, and 30,31]). It is therefore not surprising that, for various integrable equations, different specializations of Sylvester's equation show up via the universal binary Darboux transformation in the framework of bidifferential calculus (see [23, 24 and references cited there). If the "input matrices" of the Sylvester equation (in our particular case $Q$ and $Q^{\dagger}$ ) are diagonal, the solution is a Cauchy-like matrix. The generalization to non-diagonalizable input matrices, hence going beyond a "Cauchy matrix approach" (see, e.g., 32,33]), reaches solutions that are otherwise obtained via higher-order poles in the inverse scattering method or special limits of multi-soliton solutions. This generalization is of uttermost importance for our present work. 


\section{Exact solutions obtained from a simple seed solution}

A simple solution of the focusing NLS equation is given by $\mathfrak{q}_{0}=e^{2 i t} \cdot 2$ This solution is unstable (Benjamin-Feir instability), which may be regarded as the origin of the occurrence of rogue waves. A solution of (2.1) and (2.3), corresponding to $\mathfrak{q}_{0}$, is given by

$$
\phi_{0}=\left(\begin{array}{cc}
x & e^{2 \mathrm{i} t} \\
e^{-2 \mathrm{i} t} & -x
\end{array}\right) \text {. }
$$

We note that $\phi_{0, x}=J$. Writing

$$
\eta=\left(\eta_{1}, \eta_{2}\right) e^{-\mathrm{i} J t}=\left(\eta_{1} e^{-\mathrm{i} t}, \eta_{2} e^{\mathrm{i} t}\right),
$$

where $\eta_{1}$ and $\eta_{2}$ are $n$-component vectors, the linear system (2.6) takes the form

$$
\begin{aligned}
& \mathrm{i} \eta_{j, t}+Q \eta_{j, x}=0 \quad j=1,2, \\
& \eta_{1, x}+\frac{1}{2} Q \eta_{1}-\eta_{2}=0, \quad \eta_{2, x}-\frac{1}{2} Q \eta_{2}+\eta_{1}=0,
\end{aligned}
$$

which decouples to

$$
\eta_{1, x x}-\left(\frac{1}{4} Q^{2}-I\right) \eta_{1}=0, \quad \mathrm{i} \eta_{1, t}+Q \eta_{1, x}=0, \quad \eta_{2}=\frac{1}{2} Q \eta_{1}+\eta_{1, x} .
$$

Given a solution of the first two equations, we have to find a corresponding solution $\Omega$ of $(2.7)$, i.e., the rank two Lyapunov equation

$$
Q \Omega+\Omega Q^{\dagger}=\eta_{1} \eta_{1}^{\dagger}+\eta_{2} \eta_{2}^{\dagger}
$$

The solution can be written ${ }^{3}$ as $\Omega=\Omega_{1}+\Omega_{2}$, where $\Omega_{1}$ and $\Omega_{2}$ are solutions of the rank one Lyapunov equations

$$
Q \Omega_{1}+\Omega_{1} Q^{\dagger}=\eta_{1} \eta_{1}^{\dagger}, \quad Q \Omega_{2}+\Omega_{2} Q^{\dagger}=\eta_{2} \eta_{2}^{\dagger}
$$

According to Proposition 2.1.

$$
\mathfrak{q}=\left(1-\eta_{1}^{\dagger} \Omega^{-1} \eta_{2}\right) e^{2 \mathrm{i} t}
$$

is then a solution of the focusing NLS equation.

Let $Q$ now be a lower triangular Jordan block,

$$
Q=\left(\begin{array}{ccccc}
q & 0 & \cdots & \cdots & 0 \\
1 & q & \ddots & \ddots & 0 \\
0 & \ddots & \ddots & \ddots & \vdots \\
\vdots & \ddots & \ddots & \ddots & 0 \\
0 & \cdots & 0 & 1 & q
\end{array}\right)
$$

the case we are concentrating on in this work. The solution of the corresponding rank one Lyapunov equation is then given explicitly in Proposition A.1 in Appendix A. We will use an index $(k)$ to specify the matrix size. For example, $Q_{(k)}$ is the $k \times k$ matrix version of $Q$ and $\eta_{i(k)}=\left(\eta_{i 1}, \eta_{i 2}, \ldots, \eta_{i k}\right)^{\top}$. Furthermore, we set

$$
\kappa:=2 \operatorname{Re}(q), \quad \tilde{\eta}_{i, k+1}:=\eta_{i, k+1}-\kappa^{-1} \eta_{i k}, \quad k=1,2, \ldots
$$

\footnotetext{
${ }^{2}$ Applying $t \mapsto \alpha^{2} t$ and $x \mapsto \alpha x$, with a real constant $\alpha>0$, to any solution $\mathfrak{q}$ of the NLS equation, and multiplying the resulting expression by $\alpha$, yields a new solution. In this way we obtain in particular the more general solution $\alpha e^{2 \mathrm{i} \alpha^{2} t}$.

${ }^{3}$ As a consequence of 2.5 , the Lyapunov equation possesses a unique solution.
} 
Example 3.1. For $n=2$, using Proposition A.1 we obtain

$$
\Omega_{(2)}=\Omega_{(2) 1}+\Omega_{(2) 2}=\frac{1}{\kappa} \sum_{i=1}^{2}\left(\begin{array}{cc}
\left|\eta_{i 1}\right|^{2} & \eta_{i 1} \tilde{\eta}_{i 2}^{*} \\
\eta_{i 1}^{*} \tilde{\eta}_{i 2} & \left|\tilde{\eta}_{i 2}\right|^{2}+\kappa^{-2}\left|\eta_{i 1}\right|^{2}
\end{array}\right) .
$$

The determinant of $\Omega_{(2)}$ can be written in the following concise form,

$$
\operatorname{det}\left(\Omega_{(2)}\right)=\kappa^{-4}\left(\left|\eta_{11}\right|^{2}+\left|\eta_{21}\right|^{2}\right)^{2}+\kappa^{-2}\left|\operatorname{det}\left(\eta_{1}, \eta_{2}\right)\right|^{2},
$$

where $\operatorname{det}\left(\eta_{1}, \eta_{2}\right)=\eta_{11} \eta_{22}-\eta_{12} \eta_{21}$. Hence

$$
\Omega_{(2)}^{-1}=\frac{\kappa}{\operatorname{det}(\Omega)} \sum_{i=1}^{2}\left(\begin{array}{cc}
\left|\tilde{\eta}_{i 2}\right|^{2}+\kappa^{-2}\left|\eta_{i 1}\right|^{2} & -\eta_{i 1} \tilde{\eta}_{i 2}^{*} \\
-\eta_{i 1}^{*} \tilde{\eta}_{i 2} & \left|\eta_{i 1}\right|^{2}
\end{array}\right) .
$$

This leads to the following solution of the focusing NLS equation,

$$
\mathfrak{q}=\left(1-\frac{F}{\operatorname{det}\left(\Omega_{(2)}\right)}\right) e^{2 \mathrm{i} t}
$$

where

$$
F=\frac{1}{4 \operatorname{Re}(q)^{3}}\left(\eta_{21} \eta_{11}^{*}\left(\left|\eta_{11}\right|^{2}+\left|\eta_{21}\right|^{2}\right)+2 \mathrm{i} \operatorname{Re}(q) \operatorname{Im}\left[\left(\eta_{11}^{*}\right)^{2} \operatorname{det}\left(\eta_{1}, \eta_{2}\right)\right]\right),
$$

and $\eta_{1}, \eta_{2}$ have to solve the linear system (3.1). The solutions of the latter will be provided below.

From the Lyapunov equation 3.2 one finds that its solutions are nested:

$$
\Omega_{(n+1)}=\left(\begin{array}{cc}
\Omega_{(n)} & B_{(n+1)} \\
B_{(n+1)}^{\dagger} & \omega_{(n+1)}
\end{array}\right)
$$

where

$$
\begin{aligned}
& B_{(n+1)}=K^{-1}\left(\eta_{1(n)} \eta_{1, n+1}^{*}+\eta_{2(n)} \eta_{2, n+1}^{*}-\Omega_{(n)}(0, \ldots, 0,1)^{\top}\right), \\
& \omega_{(n+1)}=\frac{1}{\kappa}\left(\left|\eta_{1, n+1}\right|^{2}+\left|\eta_{2, n+1}\right|^{2}-2 \operatorname{Re}\left[(0, \ldots, 0,1) B_{(n+1)}\right]\right),
\end{aligned}
$$

and $K$ is the Jordan block $Q_{(n)}$ with $q$ replaced by $\kappa$. In order to evaluate (3.4), we need the inverse of $\Omega_{(n+1)}$. Using a well-known formula, it is given by

$$
\Omega_{(n+1)}^{-1}=\left(\begin{array}{cc}
\Omega_{(n)}^{-1}-S_{\Omega_{(n)}^{-1}}^{-1} \Omega_{(n)}^{-1} B_{(n+1)} B_{(n+1)}^{\dagger} \Omega_{(n)}^{-1} & -S_{\Omega_{(n)}^{-1}}^{-1} \Omega_{(n)}^{-1} B_{(n+1)} \\
-S_{\Omega_{(n)}^{-1}}^{-1} B_{(n+1)}^{\dagger} \Omega_{(n)}^{-1} & S_{\Omega_{(n)}^{-1}}^{-1}
\end{array}\right),
$$

with the scalar Schur complement

$$
S_{\Omega_{(n)}}=\omega_{(n+1)}-B_{(n+1)}^{\dagger} \Omega_{(n)}^{-1} B_{(n+1)} .
$$

If $\Omega_{(n)}$ and $\Omega_{(n+1)}$ are invertible, then also $S_{\Omega_{(n)}}$. The above equations allow to recursively compute the solutions of the Lyapunov equation (3.2) for $n=3,4, \ldots$, their inverses, and the corresponding NLS solution (3.4). The concrete expressions for the solutions $\eta_{1}, \eta_{2}$ of the linear system (3.1) will be provided in following subsections. 
Example 3.2. For $\Omega_{(3)}$ we obtain

$$
\begin{aligned}
& B_{(3)}=\frac{1}{\kappa} \sum_{i=1}^{2}\left(\begin{array}{c}
\eta_{i 1} \tilde{\eta}_{i 3}^{*} \\
\tilde{\eta}_{i 2} \tilde{\eta}_{i 3}^{*}+\kappa^{-2} \eta_{i 1} \tilde{\eta}_{i 2}^{*}-\kappa^{-3}\left|\eta_{i 1}\right|^{2}
\end{array}\right), \\
& \omega_{(3)}=\frac{1}{\kappa} \sum_{i=1}^{2}\left(\left|\tilde{\eta}_{i 3}\right|^{2}+\kappa^{-2}\left|\tilde{\eta}_{i 2}-\kappa^{-1} \eta_{i 1}\right|^{2}+\kappa^{-4}\left|\eta_{i 1}\right|^{2}\right) .
\end{aligned}
$$

Since $\Omega_{(2)}$ and its inverse (see $(3.6)$ ) have been elaborated in the preceding example, we can now use (3.8) to compute the inverse of $\Omega_{(3)}$ and then elaborate 3.4 .

Remark 3.3. Let $Q$ be an $n \times n$ lower triangular Jordan block. Any invertible lower triangular $n \times n$ Toeplitz matrix $A$ with constant entries commutes with it. As a consequence, $(3.4)$ is invariant under

$$
\eta_{1} \mapsto A \eta_{1}, \quad \eta_{2} \mapsto A \eta_{2}
$$

since this is a symmetry of $(3.1)$, and from 3.2 we find that $\Omega \mapsto A \Omega A^{\dagger}$.

Remark 3.4. By use of the matrix determinant lemma, (3.4) can also be expressed as

$$
\mathfrak{q}=\frac{\operatorname{det}\left(\Omega_{(n)}-\eta_{2} \eta_{1}^{\dagger}\right)}{\operatorname{det}\left(\Omega_{(n)}\right)} e^{2 \mathrm{i} t}
$$

It follows from Remark 3.3 that, if $Q$ is an $n \times n$ lower triangular Jordan block, then under a transformation of $\eta_{1}$ and $\eta_{2}$ with an invertible lower triangular Toeplitz matrix $A$ we have $\operatorname{det}\left(\Omega_{(n)}\right) \mapsto\left|a_{1}\right|^{2} \operatorname{det}\left(\Omega_{(n)}\right)$ and $\operatorname{det}\left(\Omega_{(n)}-\eta_{2} \eta_{1}^{\dagger}\right) \mapsto\left|a_{1}\right|^{2} \operatorname{det}\left(\Omega_{(n)}-\eta_{2} \eta_{1}^{\dagger}\right)$, where $a_{1}$ is the eigenvalue of $A$.

\subsection{The infinite family of Akhmediev and Kuznetsov-Ma breathers}

If $\frac{1}{4} Q^{2}-I$ is invertible, then it possesses a square $\operatorname{root}^{4} \Lambda[36]$, and the linear system $[3.1$ admits the following solutions,

$$
\eta_{1}=\cosh (\Theta) a, \quad \eta_{2}=\frac{1}{2} Q \eta_{1}+\Lambda \sinh (\Theta) a,
$$

with a constant $n$-component vector $a$ and

$$
\Theta:=\Lambda(x+\mathrm{i} Q t)+C,
$$

where $C$ is a constant $n \times n$ matrix that commutes with $\Lambda$ (and thus with $Q$ ).

Remark 3.5. The solution of (2.7), with $\eta$ given by $(3.9)$, can also be expressed as

$\Omega_{(n)}=\cosh (\Theta) X_{11} \cosh \left(\Theta^{\dagger}\right)+\cosh (\Theta) X_{12} \sinh \left(\Theta^{\dagger}\right)+\sinh (\Theta) X_{21} \cosh \left(\Theta^{\dagger}\right)+\sinh (\Theta) X_{22} \sinh \left(\Theta^{\dagger}\right)$,

where the four constant $n \times n$ matrices $X_{i j}$ have to satisfy the Lyapunov equations

$$
\begin{aligned}
& Q X_{11}+X_{11} Q^{\dagger}=a a^{\dagger}+\frac{1}{4} Q a a^{\dagger} Q^{\dagger}, \quad Q X_{22}+X_{22} Q^{\dagger}=\Lambda a a^{\dagger} \Lambda^{\dagger}, \\
& Q X_{12}+X_{12} Q^{\dagger}=\frac{1}{2} Q a a^{\dagger} \Lambda^{\dagger}, \quad Q X_{21}+X_{21} Q^{\dagger}=\frac{\mathrm{i}}{2} \Lambda a a^{\dagger} Q^{\dagger} .
\end{aligned}
$$

We observe that, if $X$ solves the rank one Lyapunov equation

$$
Q X+X Q^{\dagger}=a a^{\dagger}
$$

then $X_{11}=X+\frac{1}{4} Q X Q^{\dagger}, X_{12}=X_{21}^{\dagger}=\frac{1}{2} Q X \Lambda^{\dagger}, X_{22}=\Lambda X \Lambda^{\dagger}$, solve the preceding system.

\footnotetext{
${ }^{4}$ Another square root is $-\Lambda$, of course. We note that a matrix may possess more than two square roots (see, e.g., 34,35 ), but this is not of relevance here.
} 

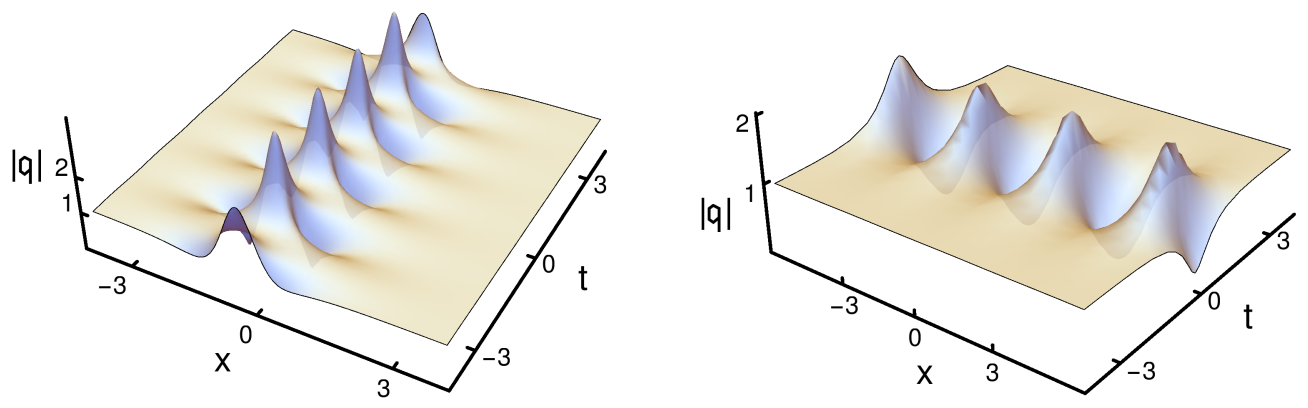

Figure 1: Plot of the modulus of the Kuznetsov-Ma breather (left plot, with $q=5 / 2$ and $c=0$ ) and the Akhmediev breather (right plot, with $q=1$ and $c=0$ ) in Section 3.1.1.

\subsubsection{Single Akhmediev and Kuznetsov-Ma breathers}

In the simplest case, $n=1$, we write $q$ instead of $Q, c$ instead of $C$, and $\lambda$ instead of $\Lambda$. From (3.2) we obtain $\Omega_{(1)}=(2 \operatorname{Re}(q))^{-1}\left(\left|\eta_{1}\right|^{2}+\left|\eta_{2}\right|^{2}\right)$, which shows that $\Omega_{(1)}$ nowhere vanishes if $a \neq 0$. We obtain the following regular solution of the focusing NLS equation,

$$
\mathfrak{q}=\left(1-\frac{1}{\Omega_{(1)}} \eta_{1}^{*} \eta_{2}\right) e^{2 \mathrm{i} t}=\left(1-4 \operatorname{Re}(q) \frac{q|\cosh (\vartheta)|^{2}+2 \lambda \cosh (\vartheta)^{*} \sinh (\vartheta)}{4|\cosh (\vartheta)|^{2}+|q \cosh (\vartheta)+2 \lambda \sinh (\vartheta)|^{2}}\right) e^{2 \mathrm{i} t},
$$

with $\lambda=\frac{1}{2} \sqrt{q^{2}-4}$ and

$$
\begin{aligned}
\vartheta:=\lambda(x+\mathrm{i} q t)+c= & \operatorname{Re}(\lambda) x-[\operatorname{Re}(\lambda) \operatorname{Im}(q)+\operatorname{Im}(\lambda) \operatorname{Re}(q)] t+\operatorname{Re}(c) \\
& +\mathrm{i}(\operatorname{Im}(\lambda) x+[\operatorname{Re}(\lambda) \operatorname{Re}(q)-\operatorname{Im}(\lambda) \operatorname{Im}(q)] t+\operatorname{Im}(c)) .
\end{aligned}
$$

As is evident from (2.7) and (2.8), the constant $a$ drops out.

If $q \neq 0$ is real and $|q|>2$, then $\lambda$ is real and $\vartheta=\lambda\left[x-x_{0}+\mathrm{i} q\left(t-t_{0}\right)\right]$, where $x_{0}=-\operatorname{Re}(c) / \lambda$ and $t_{0}=-\operatorname{Im}(c) /(\lambda q)$. The solution then becomes the Kuznetsov-Ma breather 18, 19,

$$
\mathfrak{q}=-\left(1+2 \lambda \frac{2 \lambda \cos \left(\vartheta_{1}\right)+\mathrm{i} q \sin \left(\vartheta_{1}\right)}{2 \cos \left(\vartheta_{1}\right)+2\left(1+\lambda^{2}\right) \cosh \left(\vartheta_{2}\right)+\lambda q \sinh \left(\vartheta_{2}\right)}\right) e^{2 \mathrm{i} t},
$$

where $\vartheta_{1}=2 \lambda q\left(t-t_{0}\right)$ and $\vartheta_{2}=2 \lambda\left(x-x_{0}\right)$. See Fig. 1 for a plot.

If $q \neq 0$ is real and $|q|<2$, then $\lambda$ is imaginary. In this case we write $\lambda=i \tilde{\lambda}$ with real $\tilde{\lambda}$, so that $q^{2}=4\left(1-\tilde{\lambda}^{2}\right)$. Then the above solution becomes the Akhmediev breather 57 ,

$$
\mathfrak{q}=\left(4 \tilde{\lambda} \frac{2 \tilde{\lambda} \cosh \left(\tilde{\vartheta}_{1}\right)+\mathrm{i} q \sinh \left(\tilde{\vartheta}_{1}\right)}{4 \cosh \left(\tilde{\vartheta}_{1}\right)+q^{2} \cos \left(\tilde{\vartheta}_{2}\right)-2 \tilde{\lambda} q \sin \left(\tilde{\vartheta}_{2}\right)}-1\right) e^{2 \mathrm{i} t}
$$

with $\tilde{\vartheta}_{1}=2 \tilde{\lambda} q\left(t-t_{0}\right), t_{0}=\operatorname{Re}(c) /(\tilde{\lambda} q)$, and $\tilde{\vartheta}_{2}=2 \tilde{\lambda}\left(x-x_{0}\right), x_{0}=-\operatorname{Im}(c) / \tilde{\lambda}$. See Fig. 1 for a plot.

Both, the Akhmediev and the Kuznetsov-Ma breathers, are thus special cases of the solution (3.11). The latter also contains analogs of the Kuznetsov-Ma breather with non-zero constant velocity, see Fig. 2 and also [38]. These solutions are not obtained via a Galilean transformation from those with real $q$ (cf. [39], for example). Generalizations of above solutions involving Jacobi elliptic functions appeared in 37 .

\footnotetext{
${ }^{5}$ Interpreting $t$ as time, this is actually a rogue wave which suddenly appears and then disappears. Changing the roles of $x$ and $t$ as spatial and temporal coordinates, it becomes a breather.
} 


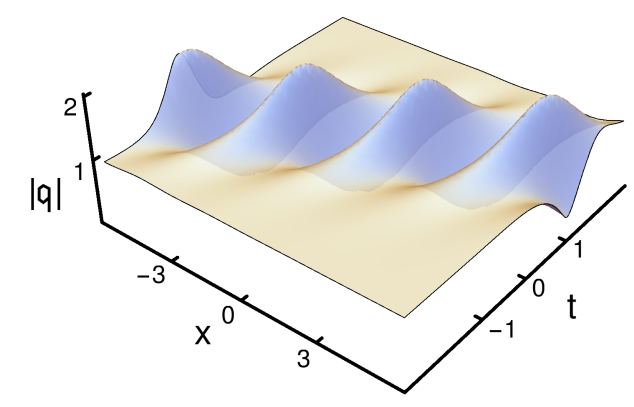

Figure 2: Plot of the modulus of the solution 3.11 in Section 3.1.1 with $c=0$ and $q=1+\mathrm{i}$.

\subsubsection{Higher order Akhmediev-Kuznetsov-Ma breathers}

Let us split the $n \times n$ lower triangular Jordan block (3.5) as

$$
Q=q I_{n}+N
$$

where $I_{n}$ denotes the $n \times n$ identity matrix and $N$ is the nilpotent matrix of degree $n$, having 1's in the second lower diagonal and otherwise zeros. For any analytic function $f$ of a single variable, we define

$$
f(Q):=\sum_{k=0}^{n-1} \frac{1}{k !} f^{(k)}(q) N^{k},
$$

using the Taylor series expansion for $f$. If $q \neq \pm 2$, then $\frac{1}{4} Q^{2}-I$ is invertible and has the square root (also see [40,41])

$$
\Lambda=f(Q) \quad \text { where } \quad f(q)=\frac{1}{2} \sqrt{q^{2}-4}
$$

This is the $n \times n$ lower triangular Toeplitz matrix

$$
\Lambda=\frac{1}{2} \sqrt{q^{2}-4}\left(\begin{array}{cccccc}
1 & 0 & \cdots & \cdots & \cdots & 0 \\
q\left(q^{2}-4\right)^{-1} & 1 & \ddots & \ddots & \ddots & \vdots \\
-2\left(q^{2}-4\right)^{-2} & \ddots & \ddots & \ddots & \ddots & \vdots \\
2 q\left(q^{2}-4\right)^{-3} & \ddots & \ddots & \ddots & \ddots & \vdots \\
\vdots & \ddots & \ddots & \ddots & \ddots & 0 \\
\vdots & & \ddots & \ddots & \ddots & 1
\end{array}\right) .
$$

Proposition 3.6. Let $Q$ be given by (3.5) with $\operatorname{Re}(q) \neq 0$ and $q \neq \pm 2$. Then the solution (3.4) of the focusing NLS equation, obtained from the solution of the linear system determined by (3.9), is regular if the first component of the vector a in (3.9) is different from zero.

Proof. $q \neq \pm 2$ is the condition for $\frac{1}{4} Q^{2}-I$ to be invertible. As a consequence of the quadratic identity for sinh and cosh (respectively, sin and cos), which is also satisfied by their matrix versions [41, $\eta_{1}$ and $\eta_{2}$ given by (3.9) cannot vanish simultaneously at any space-time point $(x, t)$ if $a_{1} \neq 0$. Now it follows from Proposition B.1 in Appendix B that the solution of the Lyapunov equation (3.2) is invertible for all $x \in \mathbb{R}$ and $t \in \mathbb{R}$. But this is the regularity condition for (3.4). 
We define the $n$-th order AKM (Akhmediev-Kuznetsov-Ma) breather as the solution of the focusing NLS equation, obtained via Proposition 2.1 with the $n \times n$ matrix $Q$ given by the Jordan block (3.5), where $\operatorname{Re}(q) \neq 0$ and $q \neq \pm 2$, and with the solution (3.9) of the linear system, where the first component of the vector $a$ is different from zero. According to Proposition 3.6, the solution is regular. The first component of $a$ can be set to 1 since it does not influence the solution determined by (3.4). Writing $a=A(1,0, \ldots, 0)^{\top}$ (with the Toeplitz matrix $A$ in A.2), Remark 3.3 shows that the parameters $a_{i}, i>1$, are redundant. Hence, without restriction of generality we can set

$$
a=(1,0, \ldots, 0) .
$$

The $n$-th order AKM breather thus depends on $n$ complex parameters. The parameter $c_{1}$ can be removed by shifts in $x$ and $t$, which are Lie point symmetries of the NLS equation.

Example 3.7. For $n=2$ we have

$$
\begin{aligned}
& Q=\left(\begin{array}{cc}
q & 0 \\
1 & q
\end{array}\right), \quad \Lambda=\left(\begin{array}{cc}
\lambda & 0 \\
\frac{q}{4 \lambda} & \lambda
\end{array}\right), \quad \Theta=\left(\begin{array}{cc}
\vartheta & 0 \\
\frac{1}{4 \lambda} \mathcal{P} & \vartheta
\end{array}\right), \\
& \eta_{1}=\left(\begin{array}{c}
\cosh (\vartheta) \\
\frac{1}{4 \lambda} \mathcal{P} \sinh (\vartheta)
\end{array}\right), \quad \eta_{2}=\left(\begin{array}{c}
\theta_{1} \\
\frac{\mathcal{P}+2}{4 \lambda} \theta_{2}
\end{array}\right),
\end{aligned}
$$

where $\lambda=\frac{1}{2} \sqrt{q^{2}-4}$,

$$
\begin{aligned}
& \vartheta=\lambda(x+\mathrm{i} q t)+c_{1}, \quad \mathcal{P}=q x+2 \mathrm{i}\left(q^{2}-2\right) t+4 \lambda c_{2}, \\
& \theta_{1}=\frac{q}{2} \cosh (\vartheta)+\lambda \sinh (\vartheta), \quad \theta_{2}=\lambda \cosh (\vartheta)+\frac{q}{2} \sinh (\vartheta),
\end{aligned}
$$

and $c_{1}, c_{2}$ are arbitrary complex constants. The determinant of $\Omega_{(2)}$ is

$$
\begin{aligned}
\operatorname{det}\left(\Omega_{(2)}\right)= & \frac{1}{(2 \operatorname{Re}(q))^{4}}\left(|\cosh (\vartheta)|^{2}+\left|\theta_{1}\right|^{2}\right)^{2}+\frac{1}{4 \operatorname{Re}(q)^{2}}\left|\frac{\mathcal{P}+2}{4 \lambda} \cosh (\vartheta) \theta_{2}-\frac{\mathcal{P}}{4 \lambda} \sinh (\vartheta) \theta_{1}\right|^{2} \\
= & \frac{1}{(2 \operatorname{Re}(q))^{4}}\left(|\cosh (\vartheta)|^{2}+\left|\frac{q}{2} \cosh (\vartheta)+\lambda \sinh (\vartheta)\right|^{2}\right)^{2} \\
& +\frac{1}{16 \operatorname{Re}(q)^{2}}\left|\mathcal{P}+2 \cosh (\vartheta)^{2}+\frac{q}{\lambda} \cosh (\vartheta) \sinh (\vartheta)\right|^{2} .
\end{aligned}
$$

The $n=2$ AKM solution of the focusing NLS equation is then given by (3.7) with

$$
\begin{aligned}
F= & \frac{1}{4 \operatorname{Re}(q)^{3}}\left[\left(|\cosh (\vartheta)|^{2}+\left|\frac{q}{2} \cosh (\vartheta)+\lambda \sinh (\vartheta)\right|^{2}\right) \cosh (\vartheta)\left(\frac{q}{2} \cosh (\vartheta)+\lambda \sinh (\vartheta)\right)\right. \\
& \left.+\frac{\mathrm{i}}{2} \operatorname{Re}(q) \operatorname{Im}\left(\mathcal{P}+2 \cosh (\vartheta)^{2}+\frac{q}{\lambda} \cosh (\vartheta) \sinh (\vartheta)\right)\right] .
\end{aligned}
$$

For real $q$ we obtain a second order Kuznetsov-Ma breather if $\lambda$ is real, and a second order Akhmediev breather if $\lambda$ is imaginary. This solution first appeared in 42]. See Fig. 3 for corresponding plots.

Example 3.8. For $n=3$ we have

$$
\begin{aligned}
& Q=\left(\begin{array}{ccc}
q & 0 & 0 \\
1 & q & 0 \\
0 & 1 & q
\end{array}\right), \quad \Lambda=\left(\begin{array}{ccc}
\lambda & 0 & 0 \\
\frac{q}{4 \lambda} & \lambda & 0 \\
-\frac{1}{8 \lambda^{3}} & \frac{q}{4 \lambda} & \lambda
\end{array}\right), \quad \quad \Theta=\left(\begin{array}{ccc}
\vartheta & 0 & 0 \\
\frac{1}{4 \lambda} \mathcal{P}_{1} & \vartheta & 0 \\
-\frac{1}{8 \lambda^{3}} \mathcal{P}_{2} & \frac{1}{4 \lambda} \mathcal{P}_{1} & \vartheta
\end{array}\right), \\
& \eta_{1}=\left(\begin{array}{c}
\cosh (\vartheta) \\
\frac{1}{32} \mathcal{P}_{1} \sinh (\vartheta) \\
\frac{1}{32 \lambda^{3}}\left(\lambda \mathcal{P}_{1}^{2} \cosh (\vartheta)-4 \mathcal{P}_{2} \sinh (\vartheta)\right)
\end{array}\right), \quad \eta_{2}=\left(\begin{array}{c}
\frac{1}{4 \lambda}\left(\mathcal{P}_{1}+2\right) \theta_{2} \\
\frac{1}{64 \lambda^{3}} \theta_{3}
\end{array}\right),
\end{aligned}
$$



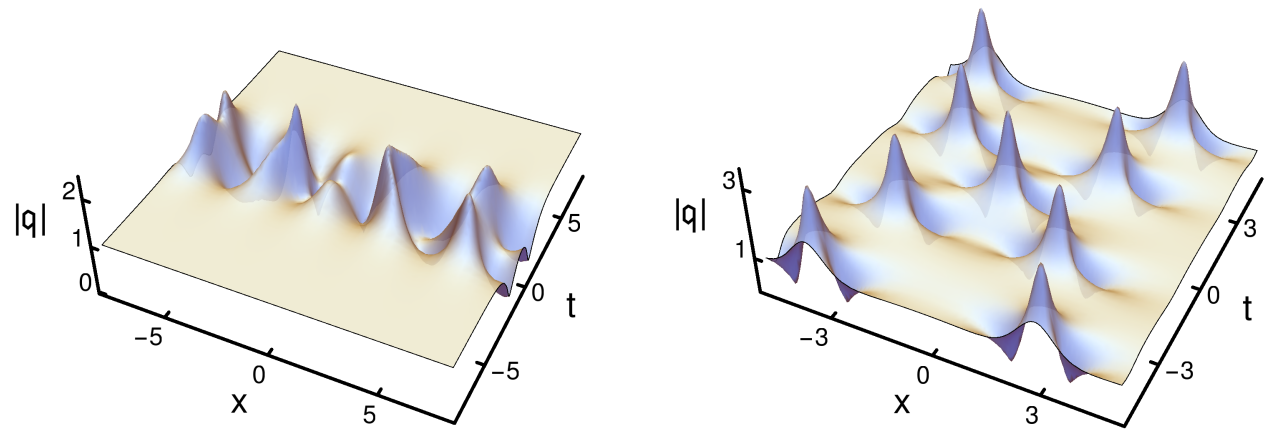

Figure 3: Plots of the modulus of the second order $(n=2)$ AKM solution in Example 3.7, with $q=1$ (a second order Akhmediev breather), respectively $q=9 / 4$ (a second order Kuznetsov-Ma breather). Here we set $c_{1}=c_{2}=0$.

where $\vartheta, \theta_{1}, \theta_{2}$ are defined as in Example 3.7 with $\mathcal{P}_{1}=\mathcal{P}$, and

$$
\begin{aligned}
& \mathcal{P}_{2}=x-\mathrm{i}\left(2 \lambda^{2}-1\right) q t-8 \lambda^{3} c_{3}, \\
& \theta_{3}=2 \lambda \mathcal{P}_{1}\left(\mathcal{P}_{1}+4\right) \theta_{1}-8 \mathcal{P}_{2} \theta_{2}-8 \sinh (\vartheta) .
\end{aligned}
$$

Inserting (3.6) and the expressions in Example 3.2 in $(3.8)$, via 3.4 we can compute the third order AKM breather. This results in a very lengthy expression and will therefore not be written explicitly.

\subsection{The family of (higher) Peregrine breathers}

Example 3.9. Let $n=1$ and $q=2$. Then the solution of the linear system (3.1) is given by $\eta_{1}=a\left[x-x_{0}+2 \mathrm{i}\left(t-t_{0}\right)\right]$ and $\eta_{2}=\eta_{1}+a$, with real constants $x_{0}, t_{0}$, and a complex constant $a$. The corresponding solution of $(3.2)$ is

$$
\Omega_{(1)}=\frac{1}{4}\left(\left|\eta_{1}\right|^{2}+\left|\eta_{2}\right|^{2}\right)=\frac{1}{8}|a|^{2}\left(16\left(t-t_{0}\right)^{2}+4\left(x-x_{0}+\frac{1}{2}\right)^{2}+1\right),
$$

which nowhere vanishes (if $a \neq 0$ ), and from 2.8 we obtain the Peregrine breather [1]

$$
\mathfrak{q}=e^{2 \mathrm{i} t}\left(1-\frac{4\left(1+4 \mathrm{i}\left(t-t_{0}\right)\right.}{16\left(t-t_{0}\right)^{2}+4\left(x-x_{0}\right)^{2}+1}\right),
$$

after a redefinition of $x_{0}$ and $\mathfrak{q} \mapsto-\mathfrak{q}$.

Now we address the case where the matrix

$$
\mathcal{N}:=\frac{1}{4} Q^{2}-I
$$

in the linear system (3.1) is degenerate. More precisely, we will concentrate on the case where $\mathcal{N}$ is nilpotent, so that zero is the only eigenvalue, which then means that the only eigenvalue of $Q$ is $q=2$.

Proposition 3.10. Let $n>0$. Let the $n \times n$ matrix $Q$ be invertible and such that $\mathcal{N}:=\frac{1}{4} Q^{2}-I$ is nilpotent of degree $n$, i.e., $\mathcal{N}^{n}=0$. Then the linear system (3.1) is solved by

$$
\begin{aligned}
\eta_{1}= & \left(Q^{-1} R_{2}(\mathcal{N}, x) R_{1}\left(-Q^{2} \mathcal{N}, t\right)+\mathrm{i} R_{1}(\mathcal{N}, x) R_{2}\left(-Q^{2} \mathcal{N}, t\right)\right) a \\
& +\left(R_{1}(\mathcal{N}, x) R_{1}\left(-Q^{2} \mathcal{N}, t\right)+\mathrm{i} Q \mathcal{N} R_{2}(\mathcal{N}, x) R_{2}\left(-Q^{2} \mathcal{N}, t\right)\right) b, \\
\eta_{2}= & \frac{1}{2} Q \eta_{1}+\eta_{1, x},
\end{aligned}
$$


where $a$ and $b$ are $n$-component constant vectors, and

$$
R_{1}(\mathcal{N}, x):=\sum_{k=0}^{n-1} \frac{x^{2 k}}{(2 k) !} \mathcal{N}^{k}, \quad R_{2}(\mathcal{N}, x):=\sum_{k=0}^{n-1} \frac{x^{2 k+1}}{(2 k+1) !} \mathcal{N}^{k} .
$$

We note that $R_{1}(\mathcal{N}, x)_{x}=\mathcal{N} R_{2}(\mathcal{N}, x)$ and $R_{2}(\mathcal{N}, x)_{x}=R_{1}(\mathcal{N}, x)$.

Proof. Expressing the first of equations (3.1) as a first order system, we obtain the solution

$$
\left(\begin{array}{c}
\eta_{1} \\
\eta_{1, x}
\end{array}\right)=\exp (S x)\left(\begin{array}{c}
g_{1} \\
g_{2}
\end{array}\right)=(\cosh (S x)+\sinh (S x))\left(\begin{array}{c}
g_{1} \\
g_{2}
\end{array}\right), \quad S:=\left(\begin{array}{cc}
0 & I \\
\mathcal{N} & 0
\end{array}\right)
$$

where $g_{i}, i=1,2$, are $n$-component vectors, only dependent on $t$. Since $\mathcal{N}$ is assumed to be nilpotent of degree $n$, using the Taylor series expansions of cosh and sinh, this yields

$$
\eta_{1}=R_{1}(\mathcal{N}, x) g_{1}+R_{2}(\mathcal{N}, x) g_{2} .
$$

The second of (3.1) now leads to

$$
g_{1, t t}+Q^{2} \mathcal{N} g_{1}=0, \quad g_{2}=-\mathrm{i} Q^{-1} g_{1, t} .
$$

The first equation is solved in the same way as we solved the first of equations (3.1):

$$
g_{1}=R_{1}\left(-Q^{2} \mathcal{N}, t\right) b+R_{2}\left(-Q^{2} \mathcal{N}, t\right) \text { i } a,
$$

with constant complex $n$-component vectors $a, b$.

Solving the Lyapunov equations (3.3), with the solutions of the linear systems given by Proposition 3.10 , then $\mathfrak{q}$ given by $(3.4)$ is a quasi-rational solution of the focusing NLS equation. The following result establishes the existence of an infinite set of regular quasi-rational solutions.

Proposition 3.11. Any solution of the focusing NLS equation obtained via Proposition 2.1 with a solution of the linear system given by Proposition 3.10, where $Q$ is the Jordan block (3.5) with eigenvalue $q=2$, is regular if the first component of the vector a is different from zero.

Proof. Since all matrices appearing in Proposition 3.10 are lower triangular, we can easily read off the first components of $\eta_{1}$ and $\eta_{2}$ :

$$
\eta_{11}=b_{1}+\left(\frac{1}{2} x+\mathrm{i} t\right) a_{1}, \quad \eta_{21}=\eta_{11}+\frac{1}{2} a_{1},
$$

where $a_{1}\left(b_{1}\right)$ is the first component of $a(b)$. Evidently, $\eta_{11}$ and $\eta_{21}$ cannot vanish simultaneously if $a_{1} \neq 0$. Now regularity follows from Proposition B.1 in Appendix B.

Multiplying $\eta_{1}$, and thus also $\eta_{2}$, by a non-zero complex constant, leaves (3.4) invariant. Therefore the first component of $a$ can be set to 1 . As a consequence of Remark 3.3 , we can set

$$
a=(1,0, \ldots, 0) .
$$

Moreover, the fact that the linear system for $\eta_{1}$ is autonomous allows us to eliminate the first component of $b$. Indeed, for any shift $x \mapsto x-x_{0}, t \mapsto t-t_{0}$, there must be a choice of the parameters that compensates it, since our solution is the general one. (3.12) with $a_{1}=1$ shows that this requires $b_{1} \mapsto b_{1}+\frac{1}{2} x_{0}+\mathrm{i} t_{0}$. Hence, without restriction of generality we may assume that

$$
b=\left(0, b_{2}, \ldots, b_{n}\right),
$$

with complex constants $b_{i}$. 

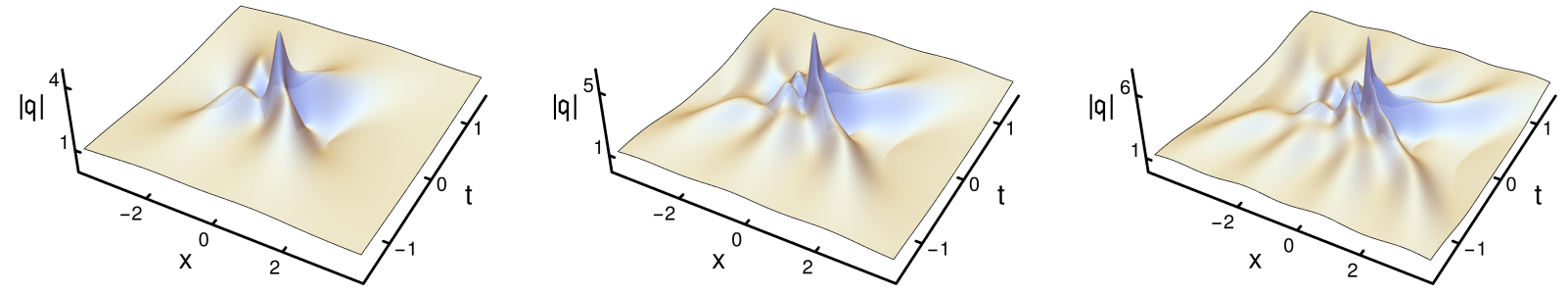

Figure 4: Plots of the modulus of the second, third and fourth order Peregrine breather solution. Here we set $b=0$.

Example 3.12. Let $n=2$ and $Q$ the $2 \times 2$ Jordan block with eigenvalue 2 . We have

$$
\eta_{1}=\left(\begin{array}{c}
\vartheta \\
\frac{2}{3} \vartheta^{3}-\frac{1}{4} x+b_{2}
\end{array}\right), \quad \eta_{2}=\left(\begin{array}{c}
\vartheta+\frac{1}{2} \\
\frac{2}{3} \vartheta^{3}+\vartheta^{2}+\frac{1}{2} \vartheta-\frac{1}{4} x+b_{2}-\frac{1}{4}
\end{array}\right),
$$

where $\vartheta=\frac{1}{2} x+\mathrm{i} t$. We obtain a quasi-rational solution of the focusing NLS equation. Replacing $x$ by $x-\frac{1}{2}$ and writing $b_{2}=\beta+\frac{1}{6}-\frac{1}{8} \mathrm{i} \alpha$, with real constants $\alpha$ and $\beta$, it can be written as

$$
\mathfrak{q}=\left(1-\frac{G+\mathrm{i} H}{D}\right) e^{2 \mathrm{i} t}
$$

where

$$
\begin{aligned}
& G=\frac{1}{3} x^{4}+\frac{1}{2} g_{2}(t) x^{2}+4 \beta x+g_{0}(t), \quad H=\frac{4}{3} t x^{4}+h_{2}(t) x^{2}+16 \beta t x+h_{0}(t), \\
& D=256 \operatorname{det}\left(\Omega_{(2)}\right)=\frac{1}{9} x^{6}+\frac{1}{12} g_{2}(t) x^{4}-\frac{4}{3} \beta x^{3}+f_{2}(t) x^{2}+\beta g_{2}(t) x+f_{0}(t), \\
& f_{0}(t)=\frac{64}{9} t^{6}+12 t^{4}-\frac{4}{3} \alpha t^{3}+\frac{11}{4} t^{2}-\frac{3}{4} \alpha t+\frac{1}{16} \alpha^{2}+4 \beta^{2}+\frac{1}{64}, \\
& g_{0}(t)=\frac{80}{3} t^{4}+6 t^{2}-\alpha t-\frac{1}{16}, \quad h_{0}(t)=\frac{64}{3} t^{5}+\frac{8}{3} t^{3}-2 \alpha t^{2}-\frac{5}{4} t+\frac{1}{8} \alpha, \\
& f_{2}(t)=\frac{16}{3} t^{4}-2 t^{2}+\alpha t+\frac{3}{16}, \quad g_{2}(t)=16 t^{2}+1, \quad h_{2}(t)=\frac{32}{3} t^{3}-2 t+\frac{1}{2} \alpha .
\end{aligned}
$$

Up to differences in notation and scalings of $\alpha$ and $\beta$, this is the second order Peregrine breather in 14. It contains the second order Peregrine solution in [2,8] as the special case $\alpha=\beta=0$.

Fig. 4 shows plots of the modulus of $\mathfrak{q}$ for the first few members of the infinite family of higher Peregrine breathers. Using computer algebra, expressions for the higher Peregrine breathers up to the tenth order have already been obtained (see [43, 44]). The role played by the parameters of the higher Peregrine breathers has been clarified in 45 47.

\subsection{Nonlinear superpositions}

If $Q=$ block-diagonal $\left(Q_{1}, \ldots, Q_{M}\right)$, then the linear system (3.1) splits into the corresponding equations for the blocks $\left\{Q_{k}\right\}$,

$$
\eta_{1, x x}^{[k]}-\left(\frac{1}{4} Q_{k}^{2}-I_{k}\right) \eta_{1}^{[k]}=0, \quad \mathrm{i} \eta_{1, t}^{[k]}+Q_{k} \eta_{1, x}^{[k]}=0, \quad \eta_{2}^{[k]}=\frac{1}{2} Q_{k} \eta_{1}^{[k]}+\eta_{1, x}^{[k]} \quad k=1, \ldots, M .
$$

Given solutions $\eta^{[k]}, k=1, \ldots, M$, all what remains to be done is to solve the Lyapunov equation with the above $Q$, and then to compute (3.4). An $n$th order AKM or Peregrine breather can be

\footnotetext{
${ }^{6}$ In all but one of the $\eta^{[k]}$, representing (higher) breathers or rogue waves, we shall now allow $a_{1}$ to be different from 1 .
} 

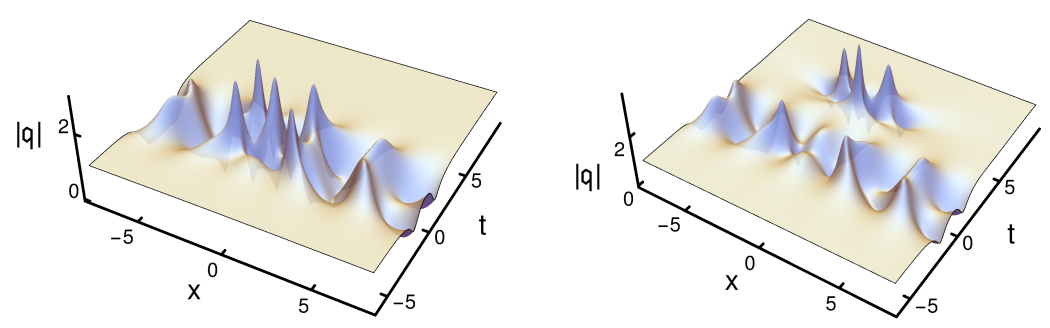

Figure 5: Plots of the modulus of $\mathfrak{q}$ for NLS solutions that are (nonlinear) superpositions of a second order Akhmediev breather (with $q=1, a_{1}=1$ and $c_{1}=c_{2}=0$ ) and a second order Peregrine breather (with $a_{1}=1, b_{1}=b_{2}=0$ and $t \mapsto t_{0}$ ). In the left plot we chose $t_{0}=0$, in the right $t_{0}=4$. In the latter case the Akhmediev breather appears at $t=0$ and (interpreting $t$ as time) essentially decays before the Peregrine breather shows up and then also decays.

obtained as a special limit of such a (nonlinear) superposition of $n$ single breathers. But, of course, one can also superpose different (higher order) breathers. Fig. 5 shows a superposition of a second order Akhmediev breather and a second order Peregrine breather. Also see [48] for other examples.

\section{Conclusions}

In this work we presented a new derivation of the families of higher order breathers and rogue waves of the focusing NLS equation, using a vectorial Darboux transformation. The latter involves a rank two Lyapunov equation where the matrix $Q$ entering its left hand side is a Jordan block. The structure of the breathers and rogue waves (including the higher order Peregrine breathers) is then essentially determined by the corresponding solution of the Lyapunov equation. The different expressions for the higher AKM and Peregrine breathers, presented in this work, should be helpful for further explorations of the structure and properties of the members of this important family of exact solutions of the focusing NLS equation.

The method presented in this work can easily be extended to vector and matrix versions of the NLS equation (also see [49 53] for different methods), its hierarchy [54], self-consistent source extensions of the NLS equation (see [24] and references cited there), and some related integrable equations. This will be addressed in forthcoming work.

Acknowledgment. The authors would like to thank Vladimir B. Matveev for some very motivating discussions about rogue waves. O.C. has been supported by an Alexander von Humboldt fellowship for postdoctoral researchers.

\section{Appendix A: Solution of the rank one Lyapunov equation with a Jordan block spectral matrix}

In this appendix we consider the rank one Lyapunov equation (see $(3.3)$, or $(3.10)$ ) with $Q$ an $n \times n$ lower triangular Jordan block (3.5), with eigenvalue $q$. If $\operatorname{Re}(q) \neq 0$, there is a unique solution and, as a consequence, $X$ is then Hermitian, i.e., $X^{\dagger}=X$. The following result is certainly known (also see, e.g., [31,55, in the context of integrable systems), though we did not find a convenient reference.

Proposition A.1. Let $a=\left(a_{1}, \ldots, a_{n}\right)^{\top}$, with $a_{i} \in \mathbb{C}$, and $\kappa:=2 \operatorname{Re}(q) \neq 0$. Then the Lyapunov 
equation

$$
Q X+X Q^{\dagger}=a a^{\dagger}
$$

with the Jordan block (3.5), has the unique solution

$$
X=\kappa A K D S D K A^{\dagger},
$$

where

$$
A=\left(\begin{array}{ccccc}
a_{1} & 0 & \cdots & \cdots & 0 \\
a_{2} & a_{1} & 0 & \cdots & 0 \\
\vdots & \ddots & \ddots & \ddots & \vdots \\
\vdots & \ddots & \ddots & \ddots & 0 \\
a_{n} & \cdots & \cdots & a_{2} & a_{1}
\end{array}\right), \quad \quad \quad \quad K=\operatorname{diag}\left(1,-1,1, \ldots,-(-1)^{n}\right)
$$

and $S$ is the $n \times n$ symmetric Pascal matrix

$$
S=\left(s_{i, j}\right), \quad s_{i, j}=\frac{(i+j-2) !}{(i-1) !(j-1) !}=\left(\begin{array}{c}
i+j-2 \\
i-1
\end{array}\right) .
$$

The determinant of $X$ is given by

$$
\operatorname{det}(X)=\kappa^{-n^{2}}\left|a_{1}\right|^{2 n} .
$$

Proof. The lower triangular Toeplitz matrix $A$ in A.2 commutes with the $n \times n$ Jordan block matrix $Q$ and achieves that $a=A(1,0, \ldots, 0)^{\top}$ (also see Lemma 6.5 in 22]). Given a solution to the special Lyapunov equation

$$
Q Y+Y Q^{\dagger}=\left(\begin{array}{cccc}
1 & 0 & \cdots & 0 \\
0 & 0 & \cdots & 0 \\
\vdots & \vdots & \vdots & \vdots \\
0 & \cdots & \cdots & 0
\end{array}\right)
$$

a solution of 3.10 is obtained as $X=A Y A^{\dagger}$. Assuming $\kappa \neq 0$, there are unique solutions $X$, respectively $Y$. The last relation implies

$$
\operatorname{det}(X)=\operatorname{det}(Y)|\operatorname{det}(A)|^{2}=\operatorname{det}(Y)\left|a_{1}\right|^{2 n} .
$$

It remains to compute $Y$ and then $\operatorname{det}(Y)$. The Lyapunov equation for $Y$ leads to linear recurrence relations. Writing

$$
Y_{i, j}=(-1)^{i+j} \kappa^{1-i-j} s_{i, j},
$$

we find that the coefficients $s_{i, j}$ are symmetric $\left(s_{i, j}=s_{j, i}\right)$ and determined by

$$
s_{1,1}=1, \quad s_{1, j}=s_{1, j-1}, \quad s_{i, j}=s_{i-1, j}+s_{i, j-1} \quad i, j=2, \ldots, n .
$$

The solution is given by

$$
s_{i, j}=\frac{(i+j-2) !}{(i-1) !(j-1) !}=\left(\begin{array}{c}
i+j-2 \\
i-1
\end{array}\right) \quad i, j=1, \ldots, n .
$$


The matrix $S=\left(s_{i, j}\right)$ is the $n \times n$ truncation of the infinite symmetric Pascal matrix (see, e.g., [56,57]). Since

$$
Y_{i, j}=\kappa(-\kappa)^{-i} s_{i, j}(-\kappa)^{-j}
$$

we have $Y=\kappa K D S D K$, and thus

$$
\operatorname{det}(Y)=\kappa^{-n^{2}} \operatorname{det}(S)=\kappa^{-n^{2}},
$$

since $\operatorname{det}(S)=1$ as an immediate consequence of the Cholesky decomposition (see, e.g., [56, 57])

$$
S=L L^{\top},
$$

with the lower triangular Pascal matrix

$$
L=\left(\ell_{i, j}\right), \quad \ell_{i, j}=\left\{\begin{array}{cl}
\left(\begin{array}{c}
i-1 \\
j-1
\end{array}\right) & \text { if } j \geq i \\
0 & \text { otherwise }
\end{array} .\right.
$$

This completes the proof of Proposition A.1.

In particular, if $a_{1} \neq 0$, the solution of the Lyapunov equation is invertible 7

\section{Appendix B: A result about the general Lyapunov equation}

In this appendix, we consider the general Lyapunov equation

$$
Q X+X Q^{\dagger}=W
$$

where $W$ is a Hermitian $n \times n$ matrix. If $Q$ and its negative Hermitian adjoint $-Q^{\dagger}$ satisfy the spectrum condition (2.5), i.e., if they have no eigenvalue in common, there is a unique solution $X$, for any Hermitian $W$. As a consequence, $X$ is then Hermitian, i.e., $X^{\dagger}=X$. The matrix $W$ can be expressed as a sum of rank one matrices (dyadic products),

$$
W=\sum_{k=1}^{m} V_{k} V_{k}^{\dagger}
$$

with linearly independent vectors $V_{k}$. Assuming the spectrum condition, we have

$$
X=\sum_{k=1}^{m} X_{k}
$$

where $X_{k}$ solves the rank one Lyapunov equation

$$
Q X_{k}+X_{k} Q^{\dagger}=V_{k} V_{k}^{\dagger}
$$

Proposition B.1. Let $Q$ be a lower triangular $n \times n$ Jordan block with eigenvalue $q$ and $\operatorname{Re}(q) \neq 0$. If the first component of one of the vectors $\left\{V_{k}\right\}$ is different from zero, then the solution of (B.1) is invertible.

Proof. Without restriction, let the first component $a_{1}$ of $a:=V_{1}$ be non-zero. Then $X_{1}$ is given by (A.1). Since a symmetric Pascal matrix is positive definite [59], (A.1) is Hermitian and positive definite if $a_{1} \neq 0$, and thus possesses a positive definite Hermitian square root. Then

$$
\operatorname{det}(X)=\operatorname{det}\left(X_{1}\right) \operatorname{det}\left(I+X_{1}^{-1 / 2}\left(\sum_{k=2}^{m} X_{k}\right) X_{1}^{-1 / 2}\right) \neq 0 .
$$

In the last step we used the fact that, according to $\mathrm{A.1}$ and $(\mathrm{A} .5)$, each $X_{k}$ has a decomposition $X_{k}=B_{k}^{\dagger} B_{k}$, with a matrix $B_{k}$, so that $X_{1}^{-1 / 2} \sum_{k=2}^{m} X_{k} X_{1}^{-1 / 2}$ is positive semi-definite.

\footnotetext{
${ }^{7}$ It is known (see [58], for example) that if $a_{1} \neq 0$, then $(Q, a)$ is controllable and the solution of the Lyapunov equation is invertible.
} 


\section{References}

[1] Peregrine D 1983 Water waves, nonlinear Schrödinger equations and their solutions J. Austral. Math. Soc. Ser. B 25 16-43

[2] Akhmediev N, Ankiewicz A and Soto-Crespo J 2009 Rogue waves and rational solutions of the nonlinear Schrödinger equation Phys. Rev. E 80026601

[3] Kibler B, Fatome J, Finot C, Millot G, Dias F, Genty G, Akhmediev N and Dudley J 2010 The Peregrine soliton in nonlinear fibre optics Nature Physics 6 790-795

[4] Chabchoub A, Hoffmann N and Akhmediev N 2011 Rogue wave observation in a water wave tank Phys. Rev. Lett. 106204502

[5] Bailung H, Sharma S and Nakamura Y 2011 Observation of Peregrine solitons in a multicomponent plasma with negative ions Phys. Rev. Lett. 107255005

[6] Bludov Y, Konotop V and Akhmediev N 2009 Matter rogue waves Phys. Rev. A 80033610

[7] Shrira V and Geogjaev V 2010 What makes the Peregrine soliton so special as a prototype of freak waves J. Eng. Math. 67 11-22

[8] Akhmediev N, Ankiewicz A and Taki M 2009 Waves that appear from nowhere and disappear without a trace Phys. Lett. A 373 675-678

[9] Chabchoub A, Hoffmann N, Onorato M and Akhmediev N 2012 Super rogue waves: Observation of a higher-order breather in water waves Phys. Rev. X 2011015

[10] Matveev V and Salle M 1991 Darboux Transformations and Solitons Springer Series in Nonlinear Dynamics (Berlin: Springer)

[11] Matveev V 2000 L.D. Faddeev's Seminar on Mathematical Physics (Advances in the Mathematical Sciences vol 201) ed Semenov-Tian-Shansky M (Providence, R.I.: AMS) pp 179-209

[12] Guo B, Ling L and Liu Q 2012 Nonlinear Schrödinger equation: Generalized Darboux transformation and rogue wave solutions Phys. Rev. E 85026607

[13] Dubard P, Gaillard P, Klein C and Matveev V 2010 On multi-rogue wave solutions of the NLS equation and positon solutions of the KdV equation Eur. Phys. J. Special Topics 185 247-258

[14] Dubard P and Matveev V 2011 Multi-rogue waves solutions of the focusing NLS equation and the KP-I equation Nat. Hazards Earth Syst. Sci. 11 667-672

[15] Gaillard P 2013 Degenerate determinant representation of solutions of the nonlinear Schrödinger equation, higher order Peregrine breathers and multi-rogue waves J. Math. Phys. 54013504

[16] Ohta Y and Yang J 2012 General high-order rogue waves and their dynamics in the nonlinear Schrödinger equation Proc. R. Soc. A 468 1716-1740

[17] Akhmediev N and Korneev V 1987 Modulation instability and periodic solutions of the Nonlinear Schrödinger equation Theor. Math. Phys. 69 1089-1093

[18] Kuznetsov E 1977 Solitons in a parametrically unstable plasma Sov. Phys. Dokl. 22 507-508

[19] Ma Y C 1979 The perturbed plane-wave solutions of the cubic Schrödinger equation Stud. Appl. Math. $6043-58$

[20] Kibler B, Fatome J, Finot C, Millot G, Genty G, Wetzel B, Akhmediev N, Dias F and Dudley J 2012 Observation of Kuznetsov-Ma soliton dynamics in optical fibre Scientific Reports 2463

[21] Chabchoub A, Kibler B, Dudley J and Akhmediev N 2014 Hydrodynamics of periodic breathers Phil. Trans. R. Soc. A 37220140005

[22] Dimakis A and Müller-Hoissen F 2010 Solutions of matrix NLS systems and their discretizations: a unified treatment Inverse Problems 26095007 
[23] Dimakis A and Müller-Hoissen F 2013 Binary Darboux transformations in bidifferential calculus and integrable reductions of vacuum Einstein equations SIGMA 9009

[24] Chvartatskyi O, Dimakis A and Müller-Hoissen F 2016 Self-consistent sources for integrable equations via deformations of binary Darboux transformations Lett. Math. Phys. 106 1139-1179

[25] Lancaster P and Tismenetsky M 1985 The Theory of Matrices (Orlando, USA: Academic Press)

[26] Horn R and Johnson C 2013 Matrix Analysis (Cambridge, UK: Cambridge University Press)

[27] Sakhnovich L 1986 Factorization problems and operator identities Russ. Math. Surv. 41 1-64

[28] Marchenko V 1988 Nonlinear Equations and Operator Algebras (Dordrecht, NL: Reidel)

[29] Sakhnovich L and Sakhnovich A and Roitberg I 2013 Inverse Problems and Nonlinear Evolution Equations (Berlin: De Gruyter)

[30] Aktosun T, Busse T, Demontis F and van der Mee C 2010 Exact solutions to the Nonlinear Schrödinger equation Operator Theory: Advances and Applications Vol. 203 (Basel: Birkhäuser) 1-12

[31] Xu D, Zhang D and Zhao S 2014 The Sylvester equation and integrable equations: I. The Korteweg-de Vries system and sine-Gordon equation J. Nonl. Math. Phys. 21 382-406

[32] Schiebold C 2010 Cauchy-type determinants and integrable systems Lin. Alg. Appl. 433 447-475

[33] Nijhoff F, Atkinson J and Hietarinta J 2009 Soliton solutions for ABS lattice equations: I. Cauchy matrix approach J. Phys. A: Math. Theor. 42404005

[34] Johnson R, Okubo K and Reams R 2001 Uniqueness of matrix square roots and an application Lin. Alg. Appl. 323 51-60

[35] Hasan M 1997 A power method for computing square roots of complex matrices J. Math. Anal. Appl. $213393-405$

[36] Cross G and Lancaster P 1974 Square roots of complex matrices Linear and Multilinear Algebra 1 289-293

[37] Akhmediev N, Eleonskii V and Kulagin N 1987 Exact first-order solutions of the nonlinear Schrödinger equation Theor. Math. Phys. 72 809-818

[38] Biondini G and Kovačič G 2014 Inverse scattering transform for the focusing nonlinear Schrödinger equation with nonzero boundary conditions J. Math. Phys. 55031506

[39] Akhmediev N, Soto-Crespo J and Ankiewicz A 2009 How to excite a rogue wave Phys. Rev. A 80043818

[40] Higham N 1987 Computing real square roots of a real matrix Linear Alg. Appl. 88-89 405-430

[41] Higham N 2008 Functions of Matrices, Theory and Computation (Philadelphia, PA, USA: SIAM)

[42] Akhmediev N, Eleonskii V and Kulagin N 1985 Generation of periodic trains of picosecond pulses in an optical fiber: exact solutions Sov. Phys. JETP 62 894-899

[43] Gaillard Pand Gastineau M 2015 The Peregrine breather of order nine and its deformations with sixteen parameters solutions to the NLS equation Phys. Lett. A 379 1309-1313

[44] Gaillard P 2015 Tenth Peregrine breather solution to the NLS equation Ann. Phys. 355 293-298

[45] Ankiewicz A, Kedziora D and Akhmediev N 2011 Rogue wave triplets Phys. Lett. A $3752782-2785$

[46] Kedziora D, Ankiewicz A and Akhmediev N 2011 Circular rogue wave clusters Phys. Rev. E 84056611

[47] Kedziora D, Ankiewicz A and Akhmediev N 2013 Classifying the hierarchy of nonlinear-Schrödingerequation rogue-wave solutions Phys. Rev. E 88013207

[48] Tajiri M and Watanabe Y 1998 Breather solutions to the focusing nonlinear Schrödinger equation Phys. Rev. E 57 3519-3519

[49] Baronio F, Degasperis A, Conforti M and Wabnitz S 2012 Solutions of the vector nonlinear Schrödinger equations: Evidence for deterministic rogue waves Phys. Rev. Lett. 109044102 
[50] Vishnu Priya N, Senthilvelan M and Lakshmanan M 2013 Akhmediev breathers, Ma solitons, and general breathers from rogue waves: A study in the Manakov system Phys. Rev. E 88022918

[51] Degasperis A and Lombardo S 2013 Rational solitons of wave resonant-interaction models Phys. Rev. E 88052914

[52] Ling L, Guo B and Zhao L C 2014 High-order rogue waves in vector nonlinear Schrödinger equations Phys. Rev. E 89 041201(R)

[53] Mu G, Qin Z and Grimshaw R 2015 Dynamics of rogue waves on a multi-soliton background in a vector nonlinear Schrödinger equation SIAM J. Appl. Math. 75 1-20

[54] Ankiewicz A, Kedziora D, Chowdury A, Bandelow U and Akhmediev N 2016 Infinite hierarchy of nonlinear Schrödinger equations and their solutions Phys. Rev. E 93012206

[55] Zhang D and Zhao S 2013 Solutions to ABS lattice equations via generalized Cauchy matrix approach Stud. Appl. Math. 131 72-103

[56] Edelman A and Strang G 2004 Pascal matrices American Math. Monthly 111 189-197

[57] Yates L 2014 Linear algebra of Pascal matrices report (student project), Math. Dept., Georgia College, Milledgeville, GA, USA, https://www. gcsu. edu/sites/files/page-assets/node-808/ attachments/yates. pdf

[58] Hearan J 1977 Nonsingular solutions of $T A-B T=C$ Lin. Alg. Appl. 16 57-63

[59] Bhatia R 2007 Positive Definite Matrices (Princeton, NJ: Princeton University Press) 